\title{
DIFFRACTION DES ÉLECTRONS LENTS
}

\author{
D. ABERDAM \\ Laboratoire de Spectrométrie Physique, \\ Université Scientifique et Médicale de Grenoble, B. P. 53, 38041 Grenoble Cedex, France
}

Résumé. - Les fondements et les possibilités d'application de la Diffraction des Electrons Lents (D. E. L.) sont passés rapidement en revue.

La géométrie de la figure de diffraction résulte de lois de conservation et de la périodicité de la surface. L'intensité des faisceaux diffractés résulte de l'interaction électron-solide et des effets de diffraction multiple.

Grâce au modèle muffin-tin, on peut séparer le problème en plusieurs parties :

- Diffusion par le potentiel des cœurs.ioniques ;

- Propagation dans un milieu à potentiel constant effectif, traduisant les interactions à $N$ corps dans le gaz d'électron ;

- Traítement de la barrière de potentiel en surface ;

- Construction du champ d'onde effectif pour le calcul des intensités.

Les possibilités d'application sont nombreuses : la D. E. L. est un puissant outil de caractérisation, sensible à l'ordre à grande distance, complément nécessaire aux méthodes d'identification chimique des atomes de surface.

En cristallographie des surfaces, les possibilités sont encore limitées aux surstructures simples. Le coût des analyses est probablement très surfait. Un bon choix de la base expérimentale devrait le rendre accessible à la plupart des laboratoires.

Abstract. - The basic ideas and possibilities of application of Low Energy Electron Diffraction (L. E. E. D.) are briefly reviewed.

The geometry of the diffraction pattern is due to conservation rules together with the periodicity of the crystal surface. The intensity of the diffracted beams is governed by the electron-solid interaction and the multiple scattering effects. The muffin-tin model allows us to split the problem into four parts :

- Scattering by ion-cores ;

- Propagation in a constant potential medium, accounting for the many body interactions of the electron with the electron gas ;

- Scattering by the surface potential barrier ;

- Self-consistent calculation of the effective wave field.

Possibilities of application are large. L. E. E. D. is a powerful method for surface characterization, sensitive to long rang order. It is a necessary complement to the methods suited for the chemical determination of the atoms on a surface, such as Auger electron spectroscopy or $\mathrm{X}$-ray photoelectron spectroscopy.

Applications to surface crystallography are still limited to simple superstructures. The analyses are far too much expensive. A suitable choice of the experimental basis should probably reduce their cost to a level manageable for many laboratories.

1. Généralités. - La diffraction des électrons lents est une technique d'étude des surfaces basée sur l'interaction d'un électron avec le potentiel périodique d'un édifice cristallin. En tant que technique de diffraction, elle est essentiellement sensible à la périodicité du potentiel, c'est-à-dire à l'ordre à longue distance, et, a contrario, peu sensible aux défauts locaux.
1. 1 LES ÉLECTRONS LENTS, SONDE DE SURFACE. - La sonde est ici une particule chargée, pour laquelle la section efficace de diffusion élastique par le potentiel cristallin est très grande devant celle de diffusion des rayons $\mathrm{X}$ par la densité électronique.

La figure 1 illustre la variation de cette section efficace totale dans le cas de l'aluminium, en fonction de 




Fig. 1. - Section efficace totale de diffusion élastique $\sigma$ de l'aluminium en fonction de l'énergie, rapportée à la maille primitive de surface $\boldsymbol{A}$.

l'énergie. On voit qu'au voisinage de son maximum, cette section efficace est du même ordre que l'aire de la maille de surface. Qui plus est, l'aluminium figure parmi les diffuseurs assez faibles.

Par ailleurs, le libre parcours moyen de collision inélastique est très petit pour un électron de faible énergie dans un cristal. Il existe de nombreux processus en cascade, qu'on peut classer en processus individuels électron-électron, ou collectifs par excitations d'ondes de plasma de volume ou de surface.

Ce libre parcours moyen $\lambda_{\mathrm{e}}$ se traduit par un temps de vie de l'électron élastique, qu'on décrit souvent par un potentiel d'absorption $V_{\text {oi }}$, partie imaginaire du potentiel optique. $\lambda_{\mathrm{e}}$ et $V_{\mathrm{oi}}$ sont liés par la relation :

$$
\lambda_{\mathrm{e}}=\left(\frac{\hbar^{2}}{2 m}\left(E-V_{\mathrm{or}}\right)\right)^{1 / 2} \frac{1}{V_{\mathrm{oi}}}
$$

où $\left(E-V_{\text {or }}\right)$ désigne l'énergie cinétique de l'électron dans le cristal.

La figure 2 illustre la variation du potentiel d'absorption et du libre parcours moyen en fonction de l'énergie. Ces courbes sont assez voisines pour beaucoup de

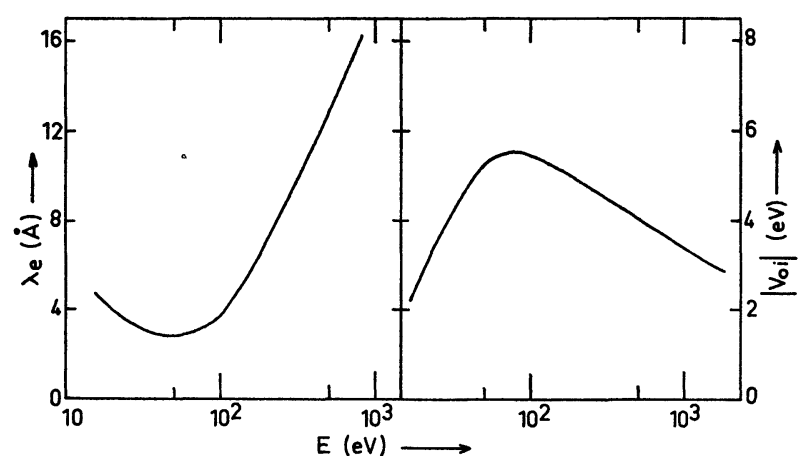

Fig. 2. - Libre parcours moyen et potentiel d'absorption en fonction de l'énergie. Ces courbes, qui présentent un certain caractère d'universalité, n'ont cependant qu'une signification semi-quantitative. matériaux, comme on peut le noter d'après les résultats compilés par Tracy et Burkstrand [1].

La conjugaison de ces deux effets : forte section efficace de diffusion élastique et très faible libre parcours moyen avant collision inélastique, limite l'épaisseur de matière susceptible de contribuer à la diffraction à quelques couches atomiques, faisant ainsi de la D. E. L. une sonde pour la structure des surfaces.

1.2 GÉOMÉTRIE DE LA FIGURE DE DIFFRACTION. La figure de diffraction est la conséquence de règles de conservation et de la périodicité de la surface du cristal.

1.2.1 Conservation de l'énergie. Dans le vide, elle s'exprime en fonction de l'énergie incidente $E$ par :

$$
\left|K_{0}\right|^{2}=\frac{2 m}{\hbar^{2}} E \text {. }
$$

La longueur d'onde correspondante est définie par:

$$
\lambda=\frac{2 \pi}{\left|K_{0}\right|} .
$$

1.2.2 Conservation de la composante de l'impulsion parallèle à la surface de l'absence de champ parallèle à la surface dans un conducteur en équilibre.

1.2.3 La faible pénétration des électrons brise la symétrie de translation du réseau perpendiculairement à la surface. Seule subsiste la périodicité latérale. Désignons par $\mathbf{g}_{\|}$les vecteurs du réseau plan réciproque du réseau de surface. La périodicité latérale implique que la conservation de $\mathbf{K}_{\|}$n'a lieu qu'à modulo $\mathbf{g}_{\|}$près. Il en découle la relation, valable dans le vide :

$K_{\perp \mathrm{g}}^{2}=\left|K_{0}\right|^{2}-\left(\mathbf{K}_{\|}+\mathbf{g}_{\|}\right)^{2}=\left|K_{0}\right|^{2}-\left|\mathbf{K}_{\| \mathbf{g}}\right|^{2}$.

$\mathbf{K}_{\| \mathbf{g}}$ et $K_{\perp \mathbf{g}}$ déterminent complètement la direction du faisceau $\mathbf{g}_{\|}$dans le vide. On désigne généralement le faisceau $\mathbf{g}_{\|}$par les deux indices de Miller $(h, k) \mathrm{du}$ vecteur $\mathbf{g}_{\|}$.

1.2.4 Dans le cristal, l'énergie diffère de l'énergie dans le vide à cause de l'existence d'un potentiel de réfraction $V_{\text {or }}$ formant un puits qui confine les électrons du solide à l'intérieur de celui-ci, et d'un potentiel d'absorption $V_{\text {oi }}$, qui traduit la courte durée de vie d'un électron d'énergie $E-V_{01}$ dans le cristal. Cet électron perd très vite de l'énergie par interaction électron-électron ou par excitation d'oscillations collectives.

Par conséquent, l'énergie dans le cristal s'écrit :

$$
\frac{\hbar^{2}}{2 m}|K|^{2}=E-V_{\text {or }}-i V_{\text {oi }} \text {. }
$$

1.3 TAILle ET FORME DES TACHES DE DIFFraCTION. - Si la surface du cristal était idéalement parfaite et le faisceau incident parfaitement cylindrique, les taches de diffraction auraient le diamètre du faisceau incident. Elles sont plus larges, pour deux raisons :

1.3.1 L'électron incident est un paquet d'ondes 
possédant une distribution en énergie, caractérisée par sa largeur relative $(\Delta E / E)$ et une distribution angulaire de largeur $\Delta \theta$ autour d'une direction d'incidence moyenne $\theta$.

L'extension spatiale et la durée de vie de l'onde plane incidente sont données par les inégalités de Heisenberg.

$$
\begin{gathered}
\Delta \text { p. } \Delta \mathbf{r}>\hbar \\
\Delta E . \Delta t>\hbar .
\end{gathered}
$$

A la surface, ces effets se combinent pour donner une longueur de cohérence $l(\alpha)$ qui dépend de la direction $\alpha$ dans laquelle elle est évaluée par rapport au plan d'incidence. Pendry [2] propose la formule :

$$
l(\alpha)=\frac{\lambda}{\left(2(\Delta \theta)^{2} \sin ^{2} \alpha+\left(\frac{\Delta E}{E}\right)^{2} \cos ^{2} \alpha\right)^{1 / 2}}
$$

$l$ est de l'ordre de quelques centaines d'angströms.

1.3.2 La surface du cristal n'est pas idéalement parfaite. Si la densité de défauts est telle que l'ordre à longue distance n'est conservé en moyenne qu'en deçàd'une distance inférieure à $l$, on observera un élargissement des taches dû à l'effet de taille des plages du cristal qui diffractent de manière cohérente. Enfin, si la répartition des défauts n'est pas isotrope sur la surface, on peut observer aussi un effet de forme, pouvant aller jusqu'à l'existence de défauts linéaires.

1.4 INTENSITÉ DES TACHES DE DIFFRACTION. - La géométrie de la figure de diffraction est d'une importance capitale car elle permet de connaître la périodicité de la surface. Ceci permet de dénombrer et de caractériser de nombreuses phases bidimensionnelles résultant de l'interaction d'une surface solide avec des gaz ou des vapeurs variés. Une grande partie des progrès accomplis dans la connaissance des surfaces résulte de cette analyse de la géométrie des figures de diffraction.

Cependant une grande quantité d'informations est contenue dans l'intensité des taches, qui est gouvernée par les propriétés de diffusion du potentiel cristallin, auxquelles se superposent les processus de diffraction multiple, très importants à cause de la grande section efficace de diffusion. Le contenu de la maille n'est donc accessible, qu'à travers le calcul de la diffraction multiple. C'est là une des sources des difficultés rencontrées en D. E. L. pour la cristallographie des surfaces. Nous allons examiner les différentes grandeurs impliquées dans l'interaction de l'électron avec le cristal dans le paragraphe suivant. Ensuite, nous discuterons divers types d'application, et en particulier, la cristallographie des surfaces.

2. Description de l'interaction d'un électron lent avec un cristal. - Cette interaction est très compliquée. Le cristal est constitué de cœurs ioniques chargés positivement, et d'électrons délocalisés dans le solide. L'interaction à $N$ électrons peut être décrite par une équation à un électron grâce à l'approximation de Hartree-Fock-Slater.

Dans cette approximation, l'électron incident interagit avec le champ coulombien des noyaux atomiques et de tous les électrons du solide. L'interaction multicorps est remplacée par un potentiel d'échange, qui trouve son origine dans le fait que les électrons sont indiscernables, qu'ils ont un spin et qu'ils obéissent à la règle d'exclusion de Pauli. Cette approximation n'est valide que dans la mesure où les états des électrons de coeurs sont stationnaires par rapport aux déplacements du noyau, et où ces déplacements sont assez petits par rapport à la maille pour que les états de cours n'en soient pas modifiés. C'est le modèle des cœurs ioniques rigides. On trouvera des références de base dans Fletcher [3].

2.1 StRUCtuRe DE BANDE OU Diffusion MULTIPLE ? - Il y a essentiellement deux approches pour la détermination de la fonction d'onde de l'électron lent : le raccordement de structure de bande et les formalismes de diffraction multiple.

En définitive, ces deux approches sont parfaitement équivalentes. Cependant, elles sont fort différentes à l'usage.

2.1.1 Le raccordement de structure de bande est l'approche la plus intuitive. Elle consiste à calculer les états propres de l'électron dans le solide (ondes de Bloch) par une méthode standard de structure de bande. Ziman [4] a récemment passé ces méthodes en revue. Habituellement, les ondes de Bloch sont développées sur une base d'ondes planes. Un tel développement ne converge bien que si le potentiel réel est remplacé par un pseudo-potentiel beaucoup plus faible. L'utilisation des pseudo-potentiels est une méthode très puissante en physique des métaux. Voir par exemple Harrison [5].

Le problème de structure de bande est résolu en cherchant des solutions pour un système d'équations semblables à celles obtenues dans l'approximation des électrons presque libres, mais avec le pseudo-potentiel.

$$
\left((\mathbf{k}-\mathbf{g})^{2}-\varepsilon\right) \alpha_{\mathbf{k}-\mathbf{z}}+\sum_{\mathbf{g}^{\prime}} \Gamma_{\mathbf{z g}} \alpha_{\mathbf{k}-\mathbf{g}^{\prime}}=0
$$

où $(\mathbf{k}-\mathbf{g})$ est le vecteur d'onde d'une onde plane dans le développement, $\&$ l'état propre de l'électron, $\alpha_{k-g}$ le coefficient de l'onde plane dans le développement et $\Gamma_{\mathbf{z z}}$ la composante de Fourier du pseudo-potentiel entre les états $\mathbf{k}-\mathbf{g}$ et $\mathbf{k}-\mathbf{g}^{\prime}$.

Ce système d'équation n'a de solution que si le déterminant séculaire s'annule. La recherche des zéros du déterminant est difficile, car le pseudo-potentiel est usuellement non local, de sorte que $\Gamma_{\mathbf{g g}}$ dépend de $\mathrm{g}$ et $\mathrm{g}^{\prime}$ séparément, et aussi de l'énergie de manière compliquée. Il faut utiliser des méthodes d'essai et erreur.

Une fois résolu le problème dans le cristal, on raccorde la fonction d'onde de l'électron dans le cristal à sa fonction d'onde dans le vide. Ce raccordement définit l'amplitude dans les différents faisceaux. 
Pendry [6] a étudié l'application de cette approche 'à la D. E. L. et a prouvé qu'elle est utilisable. Elle est cependant difficile à manipuler. Son principal inconvénient, qui l'a fait aujourd'hui presque complètement abandonner, est l'hypothèse que le cristal semi-infini possède un réseau tridimensionnel parfait. Ceci rend difficile l'étude des cas où la surface possède une structure différente de celle d'un plan parallèle en volume. Par contre elle a l'avantage de n'imposer aucune limitation au raffinement de la description du potentiel cristallin.

2.1.2 On lui préfère généralement les formalismes de diffraction multiple. Ils consistent à déterminer, de manière auto-cohérente, le champ d'onde effectif sur un site.

Ce champ d'onde résulte de la superposition du champ d'onde incident et du champ d'onde diffusé par l'ensemble des autres sites. Le champ d'onde diffusé par le site est alors proportionnel au champ d'onde effectif. Ce point de vue suppose que l'on est capable de déterminer les propriétés de diffusion d'un site et de propager le champ d'onde d'un site à l'autre. Cette distinction conceptuelle est transformée en une distinction pratique grâce à un modèle de cristal très répandu, le modèle muffin-tin, qui est aussi utilisé dans les méthodes standards de calcul de bande. En fait d'ailleurs, la structure de bande est obtenue comme un résultat secondaire du calcul en diffusion multiple, ce qui prouve bien l'équivalence fondamentale des différentes approches.

Le modèle muffin-tin consiste à séparer le potentiel cristallin en deux parties. La première contient la forte contribution des cœurs ioniques; elle est supposée avoir une symétrie sphérique et s'étendre dans une région limitée à l'intérieur d'une sphère de rayon $R_{\mathrm{m} t}$, le rayon muffin-tin tel que les potentiels de deux cœurs voisins ne se recouvrent pas. Entre les sphères, le potentiel est plat. On l'appelle le zéro muffin-tin.

La seconde contient la faible contribution du potentiel interstitiel, c'est-à-dire le potentiel entre les sphères muffin-tin. Ce potentiel est plat à l'intérieur des cœurs ioniques au niveau du zéro muffin-tin.

Il y a un certain arbitraire à choisir le rayon et le zéro muffin-tin. Le potentiel interstitiel, assez faible dans les métaux simples, peut présenter des bosses non négligeables dans des structures du type diamant. Dans les métaux simples, on le laisse généralement de côté, et le potentiel muffin-tin se réduit alors au potentiel à symétrie sphérique des cœurs, noyé dans un milieu à potentiel constant.

Ce modèle permet de réduire le problème initial à quatre problèmes plus simples :

- la diffusion par les cœurs ioniques,

- la propagation des ondes diffusées entre les cœurs ioniques,

- la spécification des conditions aux limites près de la surface,
- la construction du champ d'onde effectif sur chaque site.

Outre la simplification ainsi apportée, le modèle muffin-tin permet de construire le cristal par couches parallèles à la surface, et de traiter ainsi aisément le cas de couches de surface différentes de celles dans le volume.

Il n'est pas possible ici d'examiner en détail la manière dont on traite ces différentes étapes. C'est pourquoi je me limiterai à mentionner les idées et les hypothèses principales, ainsi que les difficultés essentielles.

2.2 DIFFÉRENTES GRANDEURS INTERVENANT DANS L'INTERACTION. - Grâce au modèle muffin-tin, nous avons vu qu'il est possible de diviser le problème en quatre parties que nous allons rapidement passer en revue.

2.2.1 Diffusion par les cæurs ioniques. - On se place dans l'approximation des cœurs ioniques rigides. Le potentiel résulte de la superposition d'une contribution de Coulomb provenant du noyau et des électrons de cœur et d'une contribution d'échange entre l'électron incident et les électrons de cœur.

La contribution coulombienne des électrons de cœur s'obtient en résolvant l'équation de Poisson, connaissant la densité électronique, calculée à partir des tables de fonctions d'ondes.

Après avoir écrit la contribution coulombienne pour un atome libre, on superpose le potentiel coulombien des autres sites du cristal. Cette superposition normalement brise la symétrie sphérique du potentiel. Mais la contribution des sites voisins d'un site donné est relativement faible à cause de l'effet d'écran des électrons non liés.

On ne fait donc pas une grosse erreur en négligeant les contributions non sphériques, afin de rester dans le modèle muffin-tin. On ajoute alors à ce potentiel coulombien la contribution d'échange. Dans l'approximation de Slater, elle est donnée par l'expression :

$$
V_{\chi}(r)=-6\left(\frac{3}{8 \pi} \rho(r)\right)^{1 / 3}
$$

Dans le cas d'une couche incomplète, on suppose que tous les sous-niveaux sont également occupés, afin de conserver la symétrie sphérique.

La construction du potentiel n'introduit aucun paramètre ajustable. Il faut maintenant choisir le rayon muffin-tin et le zéro muffin-tin. Ces deux grandeurs ne sont pas indépendantes, et ne sont pas à proprement parler des paramètres du modèle. En pratique, le seul paramètre ajustable est le zéro muffin-tin, qui précise le niveau du vide par rapport au potentiel cristallin. Nous verrons plus loin qu'on l'identifie avec le potentiel de réfraction $V_{\text {or }}$ lequel est estimé de manière indépendante.

Une fois connu le potentiel cristallin et construite sa meilleure description muffin-tin, on calcule ses propriétés de diffusion en profitant de sa symétrie sphé- 
rique. Dans ce cas en effet, l'équation de Schrödinger se sépare en une équation radiale et une équation en moments angulaires. La fonction d'ondẹ est alors développée sur une base d'harmonique sphérique. Le potentiel diffuseur agit en introduisant sur les ondes partielles des déphasages $\delta_{l}$. Ces déphasages peuvent, par exemple, être calculés par la méthode de Calogero [7]. Ils interviennent uniquement a modulo $2 \pi$. Ceci est équivalent à remplacer le potentiel par un pseudopotentiel beaucoup plus faible.

La section efficace différentielle de diffusion s'exprime en fonction des déphasages par l'équation :

$$
f(\theta, \varphi)=\frac{1}{K} \sum_{l}(2 l+1) \mathrm{e}^{i \delta_{l}} \sin \delta_{l} P_{l}(\cos \theta) .
$$

Dans cette expression, $P_{l}(\cos \theta)$ désigne les polynômes de Legendre. Les déphasages $\delta_{l}$ peuvent être renormalisés pour les effets de température, comme l'ont montré Jepsen et ses collaborateurs [8]. Ce sont alors des déphasages complexes.

2.2.2 Le potentiel optique. - Le modèle muffin-tin nous a permis de traiter séparément le problème de la diffusion par les cœurs ioniques. Il faut maintenant traiter le problème de la propagation de la fonction d'onde entre les cœurs. Le milieu interstitiel est, dans le modèle, un milieu à potentiel constant. Nous limiterons la discussion au cas des métaux simples, où le modèle muffin-tin s'applique le mieux. On doit donc déterminer le potentiel dans un milieu supposé apériodique, où se meuvent des électrons quasi libres. C'est un problème à $N$ corps, qu'on peut réduire à la détermination d'une fonction d'onde à un électron, en définissant un potentiel effectif. On utilise pour cela le modèle de jellium, discuté par Lang [9], dans lequel la charge des cœurs ioniques est distribuée uniformément dans le solide.

Le potentiel effectif, ou potentiel optique, contient toute la complexité du problème à $N$ corps. Il est le résultat de la superposition de deux contributions :

\subsubsection{Une contribution électrostatique. - Cette} contribution dans le jellium est la différence de potentiel entre deux points situés respectivement loin à l'extérieur et loin à l'intérieur du jellium semi-infini. Cette différence provient de la distribution des électrons au voisinage de la surface du solide, où la distribution de charges positives cesse brusquement. On la calcule en résolvant l'équation de Poisson, et on la note :

$$
\Delta \varphi=\varphi(-\infty)-\varphi(+\infty) .
$$

2.2.2.2 Une contribution due aux interactions à $N$ corps entre l'électron incident et les électrons quasi libres du solide.

Cette contribution est appelée la self energy de l'électron. Elle est complexe puisque l'électron incident a une durée de vie finie dans le solide. Sa partie imaginaire décrit phénoménologiquement l'absorption des électrons élastiques par le solide. Celle-ci résulte d'exci- tations réelles du gaz d'électrons, qui peuvent être individuelles (créations de paires électron-électron) et contribuent à l'absorption dès que l'énergie primaire est supérieure à l'énergie de Fermi. Elles peuvent être aussi collectives (création de plasmons), et ne contribuent à l'absorption qu'au-delà d'un seuil d'énergie nécessaire à la création de plasmon.

La partie réelle de la self energy rend compte des excitations virtuelles du gaz d'électrons, c'est-à-dire de sa polarisation élastique. Nous désignerons par le terme potentiel de réfraction le potentiel correspondant à l'énergie électrostatique dipolaire augmentée de la partie réelle de la self energy.

Le problème de la self energy a été étudié en détail par Hedin et Lundqvist [10]. Dans le modèle grossier du jellium, on trouve que le potentiel de réfraction est une fonction décroissante de l'énergie.

Pour une énergie incidente égale à l'énergie de Fermi, il est égal à la somme du potentiel électrostatique dipolaire et du potentiel d'échange-corrélation $\mu_{\mathbf{x c}}$, c'est-àdire à la somme du travail de sortie et de l'énergie de Fermi. Lorsque l'énergie incidente augmente, la contribution d'échange-corrélation diminue, et tend asymptotiquement vers zéro.

Les calculs en diffraction multiple à basse énergie et l'analyse d'expériences à moyenne énergie montrent un bon accord avec les prévisions du modèle de jellium pour l'aluminium [11].

De même, la partie imaginaire de la self energy correspond à un potentiel d'absorption. En tenant compte des interactions de paire électron-électron et de l'excitation de plasmon, on trouve, dans le jellium, un potentiel d'absorption nul à l'énergie de Fermi et un maximum pour des énergies voisines de $100 \mathrm{eV}$. Audelà, ce potentiel diminue lentement.

Le modèle, ici aussi, est en bon accord avec les résultats obtenus par l'analyse de données expérimentales [11].

Il reste à établir le lien entre le potentiel optique, estimé dans le cadre du modèle de jellium, et le zéro muffin-tin.

Ziman [4] a rapidement discuté ce problème à partir des propriétés de la méthode cellulaire pour l'étude des structures de bande. Il a montré que pour les métaux simples, le zéro muffin-tin est normalement situé en dessous du bas de la bande de conduction, mais que la différence est faible. En pratique, on le néglige et on identifie le zéro muffin-tin au potentiel de réfraction. Formellement, donc, le potentiel de réfraction et le potentiel d'absorption, paramètres ajustables du modèle, ne sont pas à l'entière disposition de l'analyste qui doit leur donner des valeurs en accord semi-quantitatif avec les prévisions faites à partir du modèle de jellium.

2.2.3 Interface cristal-vide. - Entre le vide et le cristal semi-infini parfait, il existe une région de transition, appelée en anglais selvedge. Le selvedge comprend la barrière de potentiel, constituée par le raccordement 
des potentiels de réfraction et d'absorption au potentiel du vide, et toute couche atomique ayant des propriétés géométriques, électroniques ou vibratoires différentes des propriétés de volume.

Je ne discuterai pas ici de selvedge possédant une ou plusieurs couches atomiques, mais seulement du problème de la barrière de potentiel. L'approche par raccordement de structure de bande réduit le problème à des conditions aux limites sur un plan mathématique : d'un côté le vide avec des ondes planes, de l'autre, le cristal avec des ondes de Bloch.

En fait, on sait par les équations de Maxwell que l'électron incident sent une force image en approchant du cristal, et par la physique des solides qu'il subit des interactions à $N$ corps dans le cristal. La région de la barrière de surface doit raccorder ces deux situations extrêmes, sur une distance de l'ordre de deux fois la longueur d'écrantage Thomas-Fermi.

La partie imaginaire du potentiel de surface est liée principalement à l'absorption par excitation de plasmons de surface. Elle décroît, côté vide, beaucoup plus vite que la partie réelle qui tend asymptotiquement vers le potentiel image.

Inkson [12] a étudié récemment ce problème en détail, à partir d'un modèle unidimensionnel, compatible avec l'approximation muffin-tin, puisque le potentiel de barrière doit se raccorder au potentiel optique, uniforme dans le cristal. C'est généralement une bonne approximation, sauf lorsqu'un faisceau possède un vecteur d'onde ayant une composante normale à la surface très petite. Dans ce cas, en effet, ce faisceau se propage presque parallèlement à la surface, et peut se trouver en condition de réflexion totale et sur la barrière et sur le cristal, c'est-à-dire en condition de résonance. Il est alors sensible aux faibles variations périodiques $\mathrm{du}$ potentiel interstitiel et du potentiel de barrière.

Jepsen, Marcus et Jona [8] ont calculé les intensités diffractées en choisissant différents modèles pour traiter les propriétés de la partie réelle de la barrière. Ils ont montré, qu'en général, les résultats obtenus en négligeant la réflectivité de la barrière, mais en tenant compte de la réfraction, sont meilleurs que ceux obtenus en traitant la réflexion par une barrière abrupte. Dans le domaine des basses énergies, cependant, on traite généralement la partie réelle de la barrière comme un potentiel à décroissance non abrupte (de forme exponentielle, tangente hyperbolique ou image).

La partie imaginaire est traitée approximativement en supposant qu'elle est dénuée de toute réflectivité et que le potentiel d'absorption cesse brusquement d'agir à une certaine distance de la première couche.

Les paramètres ajustables introduits dans le calcul de la barrière sont assez nombreux. Cependant, ils n'ont qu'une influence généralement faible sur les intensités calculées, sauf dans certaines conditions de résonance.

Ce sont essentiellement, pour une barrière exponentielle, la rapidité de la décroissance, la cote $z_{1}$ où débute cette décroissance, et la cote $z_{2}$ où cesse d'agir le potentiel d'absorption.
2.3 CONSTRUCTION DU CHAMP D'ONDE EFFECTIF. Il est exclu de traiter cette question en détail ici. Il existe plusieurs formąlismes de diffraction multiples, dont les plus connus sont :

- La méthode de Kambe-McRae.

- Le modèle dit de collisions inélastiques.

Ces modèles sont, en dernière analyse, équivalents.

Le plus efficace du point de vue de la souplesse et de la rapidité de calcul est certainement le premier. Examinons rapidement les idées essentielles sur lesquelles il est basé.

Dans le vide, juste au-dessus du cristal, le champ d'onde est décrit par une superposition d'ondes planes, réelles et évanescentes. Leur nombre est imposé par les conditions de convergence du développement du champ d'onde en ondes planes dans le cristal.

On définit la matrice amplitude $T_{0}$ du cristal par l'équation :

$$
b_{0}=T_{0} a_{0}
$$

où $b_{0}$ est une matrice colonne rassemblant les coefficients du développement du champ d'onde sortant dans le vide, et $a_{0}$ une matrice colonne analogue pour le champ d'onde entrant.

Calculer $T_{0}$ se fait en trois étapes, en tirant avantage du modèle muffin-tin, c'est-à-dire en découpant le cristal en couches atomiques parallèles à la surface.

a) Calcul de la matrice diffusion $S$ pour une couche.

b) Calcul de la matrice amplitude $T_{\mathrm{B}}$ pour le cristal semi-infini sans selvedge.

c) Calcul de la matrice amplitude $T_{0}$ pour le cristal habillé de son selvedge.

2.3.1 Matrice diffusion pour une couche. - Par définition, la matrice diffusion $S$ pour une couche relie le champ d'onde total émergeant au champ d'onde total incident sur la couche. Le champ d'onde incident comprend des ondes descendantes arrivant par-dessus

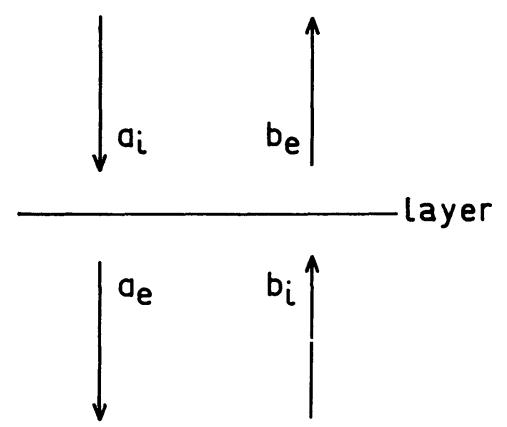

FIG. 3. - Schéma des champs d'onde incident et émergeant sur une couche. $a$ désigne une onde descendante, $b$ une onde ascendante.

la couche et des ondes montantes arrivant par-dessous la couche, et réciproquement pour le champ d'onde émergeant (Fig. 3).

$$
\left(\frac{a_{\mathrm{e}}}{b_{\mathrm{e}}}\right)=S\left(\frac{a_{\mathrm{i}}}{b_{\mathrm{i}}}\right) .
$$


Cette expression montre qu'il est commode de diviser la matrice $S$ de dimension $2 n \times 2 n$ en quatre sousblocs $n \times n$ où $n$ désigne le nombre de faisceaux inclus dans le développement du champ d'onde.

$$
S=\left(\begin{array}{ll}
S_{1} & S_{2} \\
S_{3} & S_{4}
\end{array}\right) .
$$

Le calcul de la matrice $S$ est la partie la plus difficile et la plus coûteuse du calcul. Il se fait de la manière suivante [13] :

La fonction d'onde est développée sur une base d'harmoniques sphériques à l'intérieur des sphères muffin-tin, et sur une base d'ondes planes entre les sphères muffin-tin, dans la région à potentiel constant. Les deux développements sont raccordés sur les plans supérieur et inférieur correspondant à l'épaisseur de la couche définie par le rayon muffin-tin.

2.3.2 Matrice amplitude pour le cristal sans selvedge. - Elle est obtenue à partir de la matrice diffusion $S$ d'une couche grâce à une méthode matricielle très souple proposée par McRae [14].

On définit la matrice de transfert $R$ qui relie le champ d'onde au-dessus de la couche $(N+1)$ au champ d'onde au-dessus de la couche $(N)$.

$$
\left(\begin{array}{l}
a_{\mathrm{i}}^{(N+1)} \\
b_{\mathrm{e}}^{(N+1)}
\end{array}\right)=R\left(\begin{array}{l}
a_{\mathrm{i}}^{(N)} \\
b_{\mathrm{E}}^{(N)}
\end{array}\right)
$$

et les matrices de translation $\Omega$ qui permettent de transporter le champ d'onde suivant un vecteur $\Delta \mathbf{R}$ dans la région à potentiel constant. Ces matrices ont pour éléments des termes de la forme :

$$
\omega(\mathbf{K})=\exp i(\mathbf{K} . \Delta \mathbf{R}) ;
$$

on établit aisément la relation entre $R, \Omega$ et $S$ :

$$
R=\left(\begin{array}{ll}
\Omega^{+}\left(S_{1}-S_{2} S_{4}^{-1} S_{3}\right) & \Omega^{+} S_{2} S_{4}^{-1} \\
-\Omega^{-} S_{4}^{-1} S_{3} & \Omega^{-} S_{4}^{-1}
\end{array}\right)
$$

McRae a montré que $R$ est diagonale dans une représentation du champ d'onde sur une base d'ondes de Bloch.

Donc, les valeurs propres de $R$ sont les ondes de Bloch et les vecteurs propres de $R$ sont les composantes du développement des ondes de Bloch sur une base d'ondes planes.

On voit ici le lien étroit entre le formalisme de diffraction multiple et la structure de bandes.

$\mathrm{Si}$

$$
U=\left(\begin{array}{ll}
U_{1} & U_{2} \\
U_{3} & U_{4}
\end{array}\right)
$$

est la matrice des vecteurs propres de $R$, on montre alors que $T_{\mathrm{B}}=U_{3} U_{1}^{-1}$.

Le problème du calcul des intensités, connaissant la matrice diffusion $S$ d'une couche, se ramène donc à celui de la diagonalisation de la matrice transfert $R$.
2.3.3 Matrice amplitude pour le cristal habillé par le selvedge. - Supposons que l'on connaisse la matrice diffusion $S$ pour le selvedge, défini en position par le vecteur $\Delta \mathbf{R}$, et la matrice amplitude $T_{\mathbf{B}}$ pour le cristal nu. La matrice amplitude du système total sera :

$$
\begin{aligned}
& T_{0}=S_{3}+S_{4}\left(I-\left(\Omega^{-}\right)^{-1}\right.\left.T_{\mathrm{B}} \Omega^{+} S_{2}\right)^{-1} \times \\
& \times\left(\Omega^{-}\right)^{-1} T_{\mathrm{B}} \Omega^{+} S_{1} .
\end{aligned}
$$

Cette formule est facilement généralisable au cas où le selvedge comprend une couche de périodicité latérale différente de celle des couches du cristal, à condition qu'il existe une maille de coïncidence entre les deux réseaux de surface.

Evidemment, la partie difficile du travail est le calcul de la matrice diffusion $S$ du selvedge. Cependant, l'éq. (16) met bien en évidence l'intérêt principal de ce formalisme matriciel : lorsqu'on cherche à tester différents modèles de selvedge, par exemple la position d'atomes dans une couche adsorbée formant surstructure, il n'est pas besoin de recalculer les propriétés de diffusion du volume.

2.3.4 Méthodes de calcul approché. - Différents auteurs ont essayé de réduire le coût des calculs en utilisant des méthodes approchées. Il ressort de leurs résultats que l'on ne sait pas actuellement faire de calcul approché convenable de la matrice $S$, c'est-à-dire des processus de diffraction multiple à l'intérieur d'une couche. Par contre, diverses approximations sont possibles pour la diffraction multiple entre couches. Il n'est pas possible de détailler ici ces approximations. Nous avons décrit une méthode matricielle très souple, permettant de calculer la diffraction multiple entre couches aux différents ordres successifs (1 ${ }^{\mathrm{er}}$ ordre, $2^{\mathrm{e}}$ ordre, $3^{\mathrm{e}}$ ordre, etc...), de traiter également le cas des barrières de potentiel et des couches de surstructure à partir du formalisme de Kambe-McRae. Elle donne de bons résultats et permet d'étendre le champ d'application au calcul des effets de diffraction sur des électrons résultant d'excitations diverses dans le solide [15].

3. Possibilités d'applications. - La D. E. L. est une technique relativement facile à mettre en œuvre sur le plan expérimental. Par contre, elle est difficile à exploiter sur le plan calcul. C'est pourquoi on peut distinguer sans trop d'arbitraire entre la D. E. L., outil de caractérisation physico-chimique des surfaces, et la D. E. L., technique de cristallographie de surface.

3.1 Caractérisation des SuRfaces. - La D. E. L. est une méthode pour laquelle le terme surface signifie l'ensemble des quelques toutes premières couches atomiques d'un monocristal, y compris ce qui peut se déposer dessus dans la limite de une ou deux couches. Par ailleurs, ce n'est pas une méthode de caractérisation locale, puisqu'elle est essentiellement sensible à l'ordre à grande distance. Elle permet, en pratique, de déterminer l'existence de phases solides quasi bidimensionnelles. Ce terme solide signifie ici l'existence d'un ordre à longue distance. C'est ainsi qu'on a pu montrer que la 
surface propre de certains métaux et semi-conducteurs est reconstruite (cas du silicium (111), de l'or (100), du platine (100), etc...).

De très nombreux systèmes gaz-solide ont été étudiés, en particulier l'adsorption de gaz communs (oxygène, azote, oxyde de carbone) sur les métaux (tungstène, nickel, cuivre, etc...).

Des systèmes simples tels que l'interaction d'un gaz rare sur du graphite [16] ont montré l'existence de transitions de phase bidimensionnelles du type gaz-solide. L'interaction du soufre avec de nombreux métaux a été étudiée en détail, et a montré l'existence de phases bidimensionnelles solides variées, allant jusqu'à la formation de sulfures bidimensionnels. Des diagrammes de phase ont pu être établis [17] grâce à l'étude du comportement thermique des taches de diffraction. Cependant, il faut noter que la D. E. L. ne donne que l'information relative à l'ordre à grande distance. Les autres grandeurs nécessaires à ces études sont nécessairement obtenues par d'autres voies. En particulier, la spectroscopie Auger, si elle est étalonnée par une méthode extérieure, permet la mesure des taux de recouvrement. Cette méthode a l'immense avantage qu'elle est très simple à mettre en œuvre, et qu'elle est très facile à coupler, expérimentalement, avec la D. E. L.

L'étude à basse température du comportement thermique des taches de diffraction a permis, sous réserve de certaines précautions, d'accéder à des valeurs expérimentales du déplacement carré moyen d'atomes adsorbés sur une surface [18]. En bref, la D. E. L. est un puissant outil de caractérisation, qui apporte un complément indispensable aux méthodes de détermination des espèces chimiques présentes (spectroscopie Auger, spectroscopie de photo-électrons). Cependant, il manque un maillon important de la chaîne : connaissant la nature et la quantité approximative des espèces chimiques présentes sur une surface monocristalline, connaissant l'ordre à grande distance qui s'établit, on ignore encore la distribution locale des atomes.

- Pour cela, il faudrait d'abord être capable de mesurer le degré d'homogénéité d'une distribution superficielle, à l'échelle de quelques dizaines de mailles. Pour l'instant, ceci reste, semble-t-il, un problème ouvert. Seule la microscopie à émission de champ peut apporter quelques éléments de réponse.

- Ensuite, étant sûr de l'homogénéité de la distribution, il faut connaître la distribution des atomes dans la maille de surface. C'est le problème cristallographique.

3.2 Cristallographie DES SURfaces. - Que peut-on faire avec la D. E. L. dans ce domaine ?

Prenons le cas simple d'une surstructure formée par une couche adsorbée sur une surface métallique.

Deux voies sont a priori possibles :

a) On peut tenter d'extraire, par des artifices expérimentaux (moyennes), la contribution de la diffraction simple (au $1^{\text {er }}$ ordre) du fouillis des interactions dyna- miques. Analyser ensuite ces intensités par un formalisme simple, contenant les paramètres géométriques du modèle de structure testé. La validité de cette procédure n'a jamais été démontrée. Divers auteurs l'ont appliquée et ont obtenu des résultats dont il n'a pas été possible de montrer s'ils sont bons ou non. Une discussion critique de ces méthodes, ainsi que l'étude de leur domaine de validité possible, a été faite dernièrement dans notre équipe. Il en ressort que ces méthodes peuvent avoir de l'intérêt si certaines conditions expérimentales sont respectées, mais qu'il faut rester très prudent sur les interprétations quantitatives ou semi-quantitatives qui ont été données jusqu'à présent, d'autant plus que les expériences, en général, ne satisfont pas aux conditions nécessaires de validité.

b) On peut aussi mesurer des profils d'intensité bruts d'expérience, c'est-à-dire contenant toute la complexité des effets de diffraction multiple, et tenter de calculer ces profils pour divers modèles de structure, afin de déterminer la meilleure.

Examinons rapidement quels sont les paramètres dont dispose l'expérimentateur, et quels sont les paramètres ajustables du modèle.

3.2.1 Paramètres du problème. - 3.2.1.1 L'expérimentateur peut mesurer l'intensité de divers faisceaux diffractés, en fonction de cinq paramètres :

- l'énergie $E$ du faisceau d'électrons,

- les angles d'incidence et d'azimut du faisceau d'électron, $\theta$ et $\varphi$,

- la température $T$ et l'exposition $L$, produit de la pression par le temps.

Les deux dernières variables sont supposées fixes pour une structure donnée et n'entrent donc pas en jeu. Restent donc l'énergie, l'incidence et l'azimut. La plupart des expérimentateurs, pour des raisons de commodité, mesurent des profils en énergie $I(E)$ pour plusieurs faisceaux, dans un domaine limité d'incidence $\left(0<\theta<25^{\circ}\right)$.

3.2.1.2 Du point de vue du modèle, il existe des paramètres cristallographiques, qui nous intéressent, et des paramètres non cristallographiques, qui sont des ingrédients nécessaires au calcul.

Parmi ces ingrédients, il y a :

- Les déphasages du potentiel du substrat. Ils ne sont pas ajustables.

- Les déphasages du potentiel des atomes de la couche adsorbée. Ici, le problème est plus délicat, car on peut mettre en cause la validité du modèle muffin-tin pour une telle couche. Tout dépend du type de liaison chimique. Toutefois, notons qu'il faut $a$ priori connaître la nature des atomes adsorbés.

- Le potentiel optique du substrat. Si l'étude de la surface propre a été faite soigneusement, il est logique de ne pas retoucher ces paramètres.

- Le potentiel optique dans le selvedge. En l'absence 
de donnees sur ce sujet, cela fournit deux paramètres ajustables. Cependant, la solution simple consiste à ne pas le prendre différent de celui du substrat.

- Les paramètres de décroissance et de position de la barrière de surface. Ces paramètres jouent en général un rôle mineur, et on peut leur donner une valeur réaliste à laquelle on ne touche plus.

Parmi les paramètres cristallographiques, il y a les coordonnées $x_{i} y_{i} z_{i}$ des $i$ atomes contenus dans la maille. Compte tenu de la complexité et du coût des calculs et des imperfections du modèle, il est actuellement irréaliste de traiter une couche adsorbée dont la maille contient plus d'un atome.

3.2.2 Sensibilité des expériences aux paramètres cristallographiques. - Il se pose maintenant le problème de la sensibilité des intensités mesurées aux différents paramètres, cristallographiques ou non, du modèle.

\subsubsection{Choix du domaine d'énergie. - $A$ priori,} on peut penser que la sensibilité des intensités aux propriétés de la couche de surface sera maxima lorsque la pénétration des électrons sera la plus faible; ceci indique clairement, pour le cas de l'aluminium, par exemple, que le domaine d'énergie le plus adéquat se situe entre 20 et $100 \mathrm{eV}$. En effet, c'est dans ce domaine que la section efficace de diffusion et le potentiel d'absorption sont maximums. Ceci semble accepté par l'ensemble des expérimentateurs. Un domaine reste cependant à explorer : E. G. McRae préconise de travailler à très basse énergie, en deçà du seuil d'émergence du premier faisceau non spéculaire, et de faire une analyse des intensités à partir des propriétés formelles de la fonction amplitude.

3.2.2.2 Choix des types de profils mesurés. - Par contre, il semble intéressant de critiquer les profils que les expérimentateurs relèvent et que les calculateurs calculent. En effet, comme je l'ai déjà mentionné, les gens travaillent généralement sur des profils en énergie, dans un faible domaine d'incidence.

i) Que peut-on reprocher à ces profils ?

- Ces profils sont sensibles aux interférences constructives entre plans parallèles à la surface, c'est-à-dire à un effet tridimensionnel indépendant de la couche de surface.

En pratique, ceci se traduit par de grands pics, dits enveloppes de Bragg, dont la forme, la position et la hauteur changent assez lentement avec l'incidence, pour se trouver finalement très perturbées par les effets de diffraction multiple au-delà d'environ $20^{\circ}$.

Les effets liés aux paramètres cristallographiques de la première couche apparaissent généralement comme des petites perturbations sur ces grands pics. C'est vrai pour la réflexion spéculaire. Ce peut l'être moins pour d'autres taches, relatives au substrat ou à la surstructure. Il faudra donc, en principe, relever beaucoup de ces profils, et les calculer tous pour pouvoir distinguer entre plusieurs modèles.

- Ces profils sont très onéreux à calculer, et font intervenir le plus de paramètres : en effet, il faut calculer la matrice diffusion d'une couche, qui nécessite le calcul et l'inversion d'une matrice de dimensions

$$
(2 l+1)^{2} \times(2 l+1)^{2}
$$

où $l+1$ est le nombre de déphasages nécessaires pour décrire le potentiel. Ce nombre augmente rapidement avec l'énergie. Il est de l'ordre de 6 pour l'aluminium à $100 \mathrm{eV}$, ce qui conduit déjà à des matrices $121 \times 121$. De plus, il faut diagonaliser la matrice transfert, de dimensions $2 n \times 2 n$, où $n$ est le nombre de faisceaux réels et évanescents utilisés pour développer la fonction d'onde. Ce nombre croît lui aussi rapidement avec l'énergie.

- Enfin, ils font intervenir la variation en énergie, mal connue, de paramètres comme le potentiel optique, et exigent une normalisation préalable des données expérimentales à courant incident constant.

\section{ii) Que peut-on proposer de mieux ?}

Une étude sur modèle [19] a montré l'intérêt, pour la réflexion spéculaire, de relever des cartes expérimentales d'égale intensité dans tout le domaine d'incidence. Deux types de cartes sont particulièrement utiles : les cartes en coordonnées $(E, \theta)$ et celles en coordonnées $(\theta, \varphi)$. Les relevés doivent être faits d'abord sur la surface propre, puis sur la surface avec la surstructure. Sans qu'il soit possible d'entrer dans le détail, disons simplement que ces cartes permettent de choisir les profils qui présentent les différences les plus marquantes entre le cas de la surface propre et celui de la surstructure. Elles permettent aussi, au préalable, de définir dans les meilleures conditions les paramètres non cristallographiques du substrat : potentiel optique et barrière. L'idée est simplement qu'il est préférable de rendre compte des gros effets sur de telles cartes que de détails sur des profils en énergie à faible incidence. De fait, l'expérience montre que les profils utiles sont peu nombreux mais variés. Enfin et surtout, les cartes $(\theta, \varphi)$ contiennent des profils en azimut qui ne sont sensibles qu'au changement des effets de diffraction multiple, puisque le vecteur diffusion reste constant pour ces profils.

Il s'ensuit que les cartes en $(\theta, \varphi)$ sont beaucoup plus sensibles aux propriétés de la couche de surface que les cartes $(E, \theta)$, comme on peut le voir en comparant les cartes des figures 4 et 5 .

En conclusion, disons qu'on peut espérer augmenter la confiance dans les résultats de cristallographie de surface par D. E. L., en diminuant notablement le coût de l'analyse ; celui-ci n'est à la portée que des laboratoires qui possèdent des facilités de calcul illimitées. Il avoisine en effet, étant donné la manière dont travaillent les gens actuellement, la centaine de milliers de dollars pour une structure simple. 




(a)

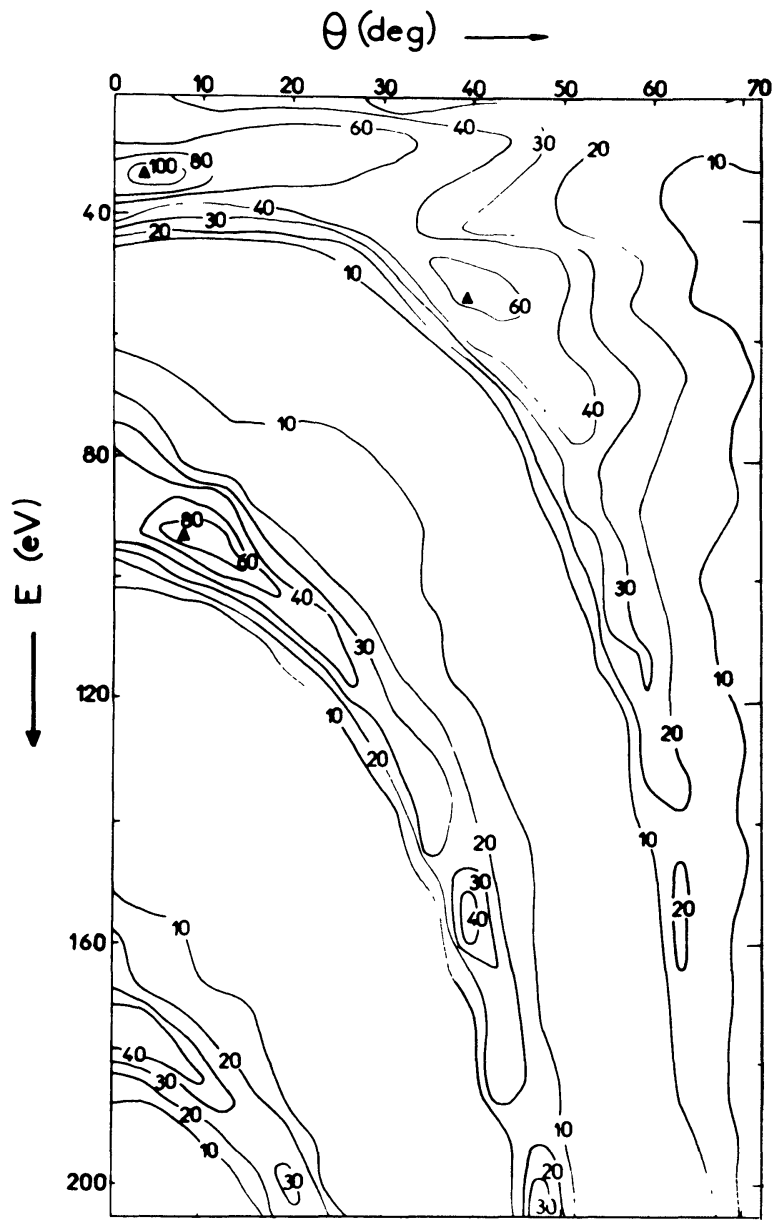

(b)

Fig. 4. - Cartes d'égale intensité pour la réflexion spéculaire, calculées en coordonnées Energie-Incidence, pour le nickel (100) propre (à gauche) et pour le nickel (100) recouvert de soufre (à droite), dans l'approximation du diffuseur isotrope.

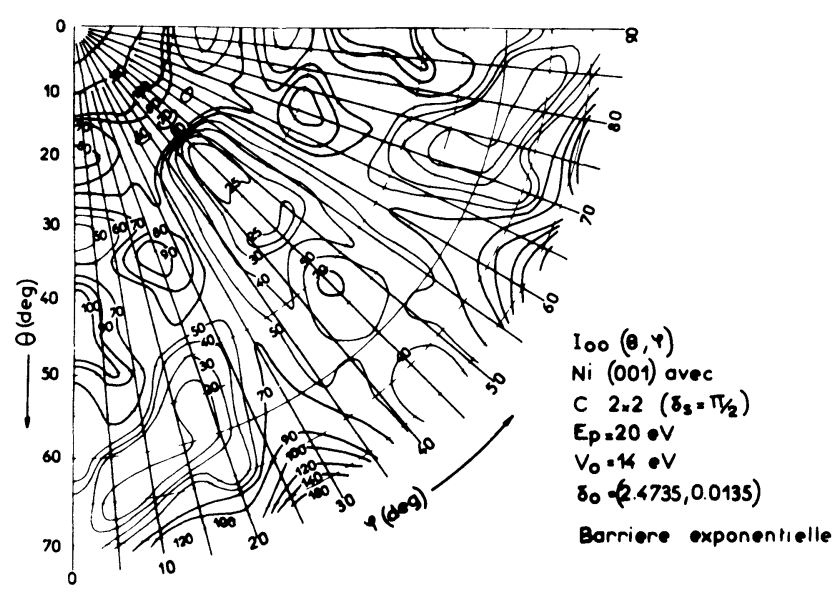

(a)



(b)

FIG. 5. - Cartes d'égale intensité pour la réflexion spéculaire dans les mêmes conditions que pour la figure 4, mais en coordonnées Incidence-Azimut.

Pour réduire ce coût, il faut diversifier la base expérimentale et choisir les meilleurs profils, grâce à la représentation des données sous forme de cartes iso-intensité. Nous pensons, de cette manière, pouvoir ramener le prix d'une analyse à la portée de la plupart des laboratoires. En contrepartie, cela exige un effort expérimental accru, mais parfaitement envisageable. Je crois que la démonstration en sera faite dans un proche avenir. 


\section{Bibliographie}

[1] Tracy, J. C. et Burkstrand, J. M., Crit. Rev. Solid State Sci. 4 (1974) 380.

[2] Pendry, B. J., Low Energy Electron Diffraction. Techniques of Physics Series, $\mathrm{n}^{\circ} 2$ (Acad. Press. N. York, London) 1974.

[3] Fletcher, G. C., The Electron Band Theory of Solids (North Holland. Amsterdam, London) 1971.

[4] Ziman, J. M., Solid State Phys. 26 (1971) 1.

[5] HARrison, W. A., Pseudopotentials in the theory of metals (Benjamin inc. B. Y., Amsterdam) 1966

[6] Pendry, J. B., J. Phys. C 2 (1969), 1215, 2273, 2283.

[7] Calogero, F., Variable phase approach to potential scattering (Acad. Press) 1967.

[8] Jepsen, D. W., Marcus, P. M. et Jona, F., Phys. Rev. B 5 (1972) 3933.

[9] Lang, N. D., Solid State Phys. 28 (1973) 225.

[10] Hedin, L. et LundQvist, S., Solid State Phys. 23 (1969) 1.
[11] Aberdam, D., Baudoing, R., Gaubert, C. et McRae, E. G., A L. E. E. D. Data reduction method. Submitted to Surf. Sci.

[12] Inkson, J. C., Surf. Sci. 28 (1971) 69 and lectures notes of the 6th L. E. E. D. seminar. Washington D. C. (1972).

[13] KAMBE, K., Z. Naturforsch. a 22 (1967) 322; 22 (1967) 422; 23 (1968) 1250.

[14] McRae, E. G., Surf. Sci. 11 (1968) 479.

[15] Groupe d'étude des surfaces, Surf. Sci. 48 (1975) 509.

[16] Suzanne, J., Coulomb, J. P. et Bienfait, M., Surf. Sci. 44 (1974) 41.

[17] Kostelitz, M., Domange, J. L. et Oudar, J., Surf. Sci. 34 (1973) 431.

[18] Theeten, J. B., Domange, J. L. et Hurault, J. P., Surf. Sci. 35 (1973) 145 et L. E. E. D.-7 Seminar notes, APS Meeting San Diego (California) 1973.

[19] Groupe D'Étude des SURfaCes, Surf. Sci. 48 (1975) 497. 